\title{
Adaptación cultural y validación del índice de discapacidad vocal pediátrico al español chileno
}

\section{Cultural adaptation and validation of the pediatric vocal handicap index to chilean spanish}

\author{
Diana Centeno A. ${ }^{1}$, Loreto Nercelles C. ${ }^{2,3}$, Christopher Catalán DV. ${ }^{1}$, Juan Valenzuela $F^{1}$
}

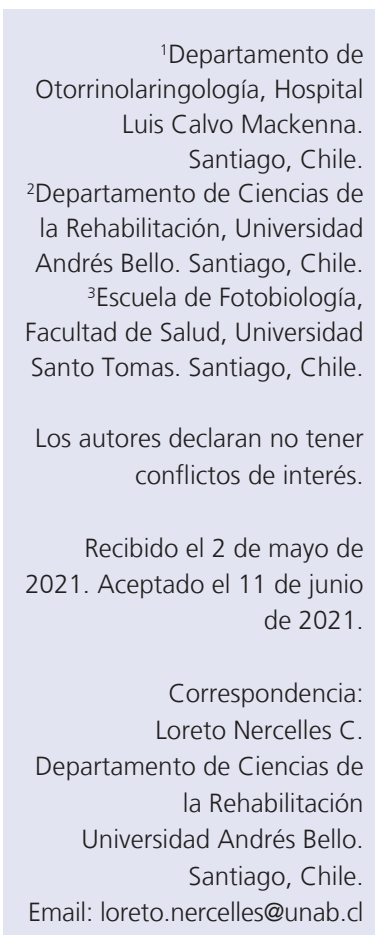

Email: loreto.nercelles@unab.cl

\section{Resumen}

Introducción: La prevalencia de patología vocal en niños fluctúa entre el 6\% y el 23\%. El cuestionario Pediatric Voice Handicap Index (pVHI) se ha transformado en el instrumento más utilizado a nivel internacional para determinar el impacto de la disfonía en la calidad de vida de los niños. Objetivo: Realizar la traducción, adaptación cultural y validación del Pediatric Voice Handicap Index al español chileno. Material y Método: Estudio transversal que incluyó a 151 niños y niñas entre 3 y 15 años. Se siguieron los 5 pasos recomendados por Beaton. La versión original del pVHI en inglés fue traducida al español por dos traductoras. Posteriormente, la versión traducida y unificada fue revisada por una lingüista chilena quien realizó la adaptación cultural al idioma español chileno. El cuestionario se aplicó a dos grupos de estudio, un grupo de niños con disfonía $(\mathrm{n}=51)$ y un grupo control de niños sin alteraciones de la voz $(\mathrm{n}=100)$ para obtener la versión final. Resultados: Se encontraron diferencias significativas entre el grupo de niños con disfonía y el grupo control en la puntuación global del pVHI y las diferentes subescalas $(\mathrm{p}<0,001)$. Se encontró una consistencia interna óptima con un excelente alfa de Cronbach $(\alpha=0,93)$, con una alta fiabilidad test-retest (puntuación de correlación de Pearson $=0,95)$. Conclusión: La versión chilena del cuestionario pVHI presenta un alto grado de validez y confiabilidad. Recomendamos su uso e implementación como protocolo estándar en la evaluación y seguimiento de la voz pediátrica. Palabras clave: Voz infantil, disfonía infantil, índice de discapacidad vocal pediátrico.

\begin{abstract}
Introduction: The prevalence of vocal pathology in children fluctuates between $6 \%$ and $23 \%$. The pediatric voice handicap index ( $p V H I$ ) questionnaire has become the most widely used instrument to determine the impact of dysphonia on the quality of life of children. Aim: Perform the translation, cultural adaptation, and validation of the pediatric voice handicap index into Chilean Spanish. Material and Method: Cross-sectional study that included 151 boys and girls between 3 and 15 years old. The 5 steps recommended by Beaton were followed. The original version of the pVHI in English was translated into Spanish by two translators. Subsequently, the translated and unified version was reviewed by a Chilean linguist who made the cultural adaptation to the Chilean Spanish language. The questionnaire was applied to two study groups, a group of children with dysphonia $(n=51)$ and a control group of children without voice disorders $(n=100)$ to obtain the final version. Results: Significant differences were found between the group of children with dysphonia and the control group in the global $p$ VHI score and the different subscales $(p<0.001)$. Optimal internal consistency was found with excellent Cronbach's alpha $(\alpha=0.93)$, with high test-retest reliability (Pearson's correlation score $=0.95$ ). Conclusion: The Chilean version of the $p V H I$ questionnaire presents a high degree of validity and reliability. We recommend its use and implementation as a standard protocol in the evaluation and monitoring of pediatric voice.
\end{abstract}

Keywords: pediatric voice, pediatric dysphonia, pediatric vocal disability index. 


\section{Introducción}

La voz es un instrumento de vital importancia para el ser humano, considerándose uno de los primeros signos de vida. La disfonía corresponde a la alteración de alguno de los parámetros acústicos de la voz como el tono, la intensidad o el timbre ${ }^{1-3}$. La prevalencia de la patología vocal en niños fluctúa entre $6 \%$ al $23 \%$ de la población infantil, dependiendo de la región geográfica en estudio y la metodología de recolección de datos utilizada, siendo más prevalente en el género masculino ${ }^{4-7}$. El proceso de evaluación de las alteraciones vocales en los niños consiste en una anamnesis exhaustiva, exploración laringológica mediante videoestroboscopía transnasal flexible o transoral rígida, valoración clínica perceptual de la voz, análisis acústico y escalas de percepción del handicap vocal ${ }^{3,4,8-12}$.

El cuestionario pediatric voice handicap index (pVHI) se ha transformado en el instrumento más utilizado a nivel internacional para determinar el impacto de la disfonía en la calidad de vida de los niños. El pVHI es un cuestionario confiable de fácil utilización en la práctica diaria. Consta de 23 preguntas, aplicadas a los padres y/o cuidadores para identificar su percepción acerca del trastorno vocal del niño o niña. El cuestionario evalúa el impacto de los trastornos de la voz a nivel funcional, físico y emocional. Cada una de las preguntas requiere una respuesta basada en la frecuencia con la que los padres y/o cuidadores percibieron cada acontecimiento. Cada respuesta se califica individualmente en una escala de 5 puntos, que va desde "nunca" (puntuación 0) hasta "siempre" (puntuación 4), con un rango de puntuación total que va de 0 a 92. También consta de una escala analógica visual al comienzo de la prueba para clasificar la locuacidad del niño. En base a los resultados de Sanz y cols., se consideran patológicas las puntuaciones de $24 \pm 11^{13-17}$.

El uso del pVHI es ampliamente recomendado por diversos autores como protocolo estándar esencial en la evaluación vocal pediátrica $^{18-21}$. En la actualidad, las versiones disponibles al español son las realizadas por Sanz y cols. en el año 2015 y la versión argentina por Carrera y cols. en el año 2019 ${ }^{17,22}$. Sin embargo, y pese a compartir el mismo idioma con estos países, se hace necesaria una validación y adaptación cultural del pVHI al español chileno para lograr una correcta interpretación de las preguntas del cuestionario.

\section{Objetivo}

El objetivo de este estudio es realizar la traducción, adaptación cultural y validación del pediatric voice handicap index al español chileno.

\section{Material y Método}

\section{Diseño}

Se realizó un estudio transversal en el Hospital de Niños Dr. Luis Calvo Mackenna en Santiago de Chile entre marzo de 2019 y marzo de 2020. Este estudio fue aprobado por el Comité de Ética del Hospital. Los procedimientos del estudio no presentan conflictos éticos y fueron elaborados según los principios de la Declaración de Helsinki. En el estudio participaron un total de 151 padres o cuidadores de niños los cuales debían tener nociones básicas de lectoescritura. Entre los encuestados, 51 eran padres/cuidadores de niños con patología de la voz (grupo experimental), y 100 eran padres/cuidadores de niños sin patología vocal (grupo control). Los niños con patología vocal fueron evaluados y diagnosticados por el equipo de la Unidad de Voz del Hospital de Niños Dr. Luis Calvo Mackenna. Los niños del grupo control no reportaron antecedentes actuales o pasados de patología vocal, así como tampoco cualquier queja de su voz, pérdida de audición o cualquier otra dificultad que pudiera afectar el habla.

\section{Desarrollo de la versión chilena del pVHI}

Para llevar a cabo la validación y adaptación cultural, se siguieron los 5 pasos recomendados por Beaton ${ }^{23}$.

\section{Paso 1}

La versión original del pVHI en inglés realizada por Zur y cols., en el año 2007, fue traducida al español por dos traductoras nativas de habla hispana: una traductora sin conocimiento del tema y una traductora especializada 
en traducción científica y con conocimiento del tema. Cada traductora emitió su reporte de manera independiente.

\section{Paso 2}

Se realizó la comparación de ambas traducciones y síntesis mediante el análisis y resolución de discrepancias en caso de existir. Elaboración de una versión unificada. La versión traducida y unificada fue revisada por una lingüista chilena, cuya misión fue realizar la adaptación cultural al idioma español chileno de la versión entregada por las traductoras.

\section{Paso 3}

La versión adaptada culturalmente fue entregada a dos personas nativas de habla inglesa para realizar una traducción inversa y poder corroborar los ítems con la versión original.

\section{Paso 4}

La versión chilena final fue creada luego de un consenso final entre los traductores, la lingüista y los autores principales de la investigación.

\section{Paso 5}

Finalmente, el cuestionario fue sometido a una prueba de pilotaje a 20 padres o cuidadores para evaluar la comprensión de cada pregunta realizándose las modificaciones pertinentes para su mejor comprensión. La Figura 1 muestra la versión en español chileno del pVHI.

\section{Aplicación del pVHI versión español chileno}

En primera instancia, los padres/cuidadores fueron contactados telefónicamente donde se les entregó las instrucciones para completar el cuestionario que posteriormente fue enviado en formato formulario google. Para el análisis de fiabilidad test-retest de pVHI, el pVHI chileno se completó dos veces con un intervalo de aproximadamente 2 semanas entre cada administración por parte de los padres/cuidadores de los niños con disfonía y por parte de los padres/cuidadores de los niños controles. Se seleccionó este intervalo porque no se esperaba que se produjera ningún cambio sustancial en la condición de la voz de los niños durante este período. Cuando los padres/cuidadores completaron el segundo pVHI, no tuvieron acceso a sus respuestas del primer $\mathrm{pVHI}$. La fiabilidad test-retest se evaluó para la puntuación total, así como para cada una de las tres subescalas de pVHI.

\section{Análisis estadístico}

El análisis estadístico se realizó con SPSS 16.0. Se utilizó la prueba U de Mann-Whitney no paramétrica para evaluar las diferencias entre los grupos control y experimental para todas las subescalas, así como las puntuaciones totales. Se utilizó alfa de Cronbach como medida de la consistencia interna. Un valor superior a 0,9 se consideró excelente. La confiabilidad del test y retest se evaluó determinando el nivel de concordancia entre los resultados del primer y segundo $\mathrm{pVHI}$ con el coeficiente de correlación de Pearson. Un valor entre 0,41 y 0,60 se consideró "moderado", un valor entre 0,61 y 0,80 se consideró "sustancial" y un valor de 0,81 se consideró "casi perfecto".

\section{Resultados}

Del total de 151 pacientes entre 3 y 15 años (media $=9,07, \mathrm{DE}=3,68$ ), 95 fueron niños y 56 fueron niñas. Las características demográficas de la muestra se presentan en la Tabla 1. Las patologías encontradas en el grupo experimental se detallan en la Tabla 2. La puntuación total y de las subescalas física, emocional y funcional del pVHI en la versión chilena se muestran en la Tabla 3.

Existieron diferencias estadísticas entre los grupos de estudio en la puntuación media total del pVHI y la puntuación media para cada ítem funcional, físico y emocional. Los puntajes de severidad del grupo experimental mostraron los puntajes más altos para la subescala física, seguida de las escalas funcional y emocional. La consistencia interna del cuestionario se determinó mediante el coeficiente alfa de Cronbach. El valor general del cuestionario obtenido de los 151 padres o cuidadores fue extremadamente alto $(\alpha=0,931)$, así como el valor de las subescalas: física $=0,925$, funcional $\alpha=0,912$ y emocional $\alpha=0,943$ (Tabla 4 ). 


\section{INDICE DE DISCAPACIDAD VOCAL PEDIATRICO ESPAÑOL CHILENO}

Con qué nota calificaría la locuacidad (cantidad que habla) de su hijo/a. Marque con un círculo:

$\begin{array}{ccccccc}1 & 2 & 3 & 4 & 5 & 6 & 7 \\ \begin{array}{c}\text { Nada } \\ \text { conversador }\end{array} & & & \begin{array}{c}\text { Moderadamente } \\ \text { conversador }\end{array} & & & \begin{array}{c}\text { Extremadamente } \\ \text { conversador }\end{array}\end{array}$

\section{Instrucciones:}

Estas son afirmaciones que muchas personas utilizan para describir la voz de sus hijos/as y cómo afectan sus vidas. Marque la respuesta de cuán frecuentemente Ud. tiene la misma experiencia, siendo:

\section{$\begin{array}{lll}0=\text { Nunca } & \text { 1 = Casi nunca } \quad 3=\text { A veces } & \text { Casi siempre }\end{array}$}

\section{Dimensión Funcional}

1) La voz de mi hijo/a es difícil de ser escuchada por las personas.

2) Las personas tienen dificultad para entender a mi hijo/a en lugares ruidosos.

$\begin{array}{lllll}0 & 1 & 2 & 3 & 4\end{array}$

3) Tenemos dificultad para escuchar a nuestro hijo/a cuando nos llama dentro de la casa o desde otra habitación.

4) Mi hijo/a tiende a evitar comunicarse con las personas debido a su voz.

5) Mi hijo/a habla con menos frecuencia con amigos, vecinos o familiares debido a su

voz.

6) Las personas le piden a mi hijo/a que repita lo que dice cuando está hablando frente a frente.

7) Las dificultades en la voz de mi hijo/a limitan sus actividades personales, educativas y sociales.

\section{Dimensión Física}

1) Mi hijo/a se queda sin aire cuando está hablando. $\quad 0 \quad 1 \quad 2 \quad 3 \quad 4$

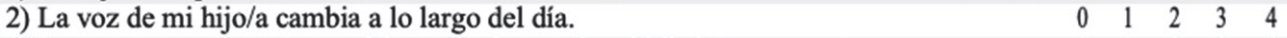

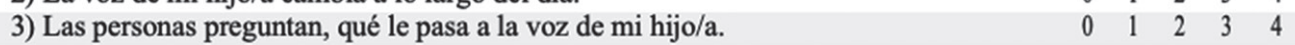

4) La voz de mi hijo/a suena seca, áspera y/o ronca. $\quad \begin{array}{lllll} & 0 & 2 & 3 & 4\end{array}$

5) La calidad de la voz de mi hijo/a es impredecible, no sabemos qué pasará con su $\quad \begin{array}{lllll}0 & 1 & 2 & 3 & 4\end{array}$ voz.

6) $\mathrm{Mi}$ hijo/a requiere de un gran esfuerzo para hablar.

7) La voz de mi hijo/a empeora en la tarde.

8) La voz de mi hijo/a se debilita o apaga al hablar.

9) $\mathrm{Mi}$ hijo/a tiene que gritar para que otros lo/la escuchen.

$\begin{array}{lllll}0 & 1 & 2 & 3 & 4 \\ 0 & 1 & 2 & 3 & 4 \\ 0 & 1 & 2 & 3 & 4 \\ 0 & 1 & 2 & 3 & 4\end{array}$

\section{Dimensión Emocional}

1) Mi hijo/a se ve tenso/a cuando habla con otras personas debido a su voz.

2) Las personas parecen incomodarse con la voz de mi hijo/a.

3) Encuentro que las personas no comprenden el problema que tiene mi hijo/a en su voz.

4) Mi hijo/a se frustra por su problema en la voz.

5) Mi hijo/a es más introvertido debido al problema de su voz.

6) Mi hijo/a se molesta cuando las personas le piden que repita lo que dijo.

7) $\mathrm{Mi} \mathrm{hijo/a} \mathrm{se} \mathrm{avergüenza} \mathrm{cuando} \mathrm{le} \mathrm{piden} \mathrm{que} \mathrm{repita} \mathrm{lo} \mathrm{que} \mathrm{dijo.}$

$\begin{array}{lllll}0 & 1 & 2 & 3 & 4 \\ 0 & 1 & 2 & 3 & 4 \\ 0 & 1 & 2 & 3 & 4 \\ 0 & 1 & 2 & 3 & 4 \\ 0 & 1 & 2 & 3 & 4 \\ 0 & 1 & 2 & 3 & 4 \\ 0 & 1 & 2 & 3 & 4\end{array}$

\section{Para ser llenado por el equipo}

Funcional $\square$ Física $\square$ Emocional $\square$ Total $\square$ Locuacidad $\square$ 
ARTíCULO DE INVESTIGACIÓN

Tabla 1. Características demográficas de los grupos control y experimental

\begin{tabular}{lccccc}
\hline & $\mathbf{n}$ & Niños & Edad promedio & Niñas & Edad promedio \\
Grupo control & 100 & 58 & 9,01 & 42 & 9,24 \\
Grupo experimental & 51 & 37 & 9,24 & 14 & 9,43 \\
Total & 151 & 95 & 9,12 & 56 & 9,33 \\
\hline
\end{tabular}

\section{Tabla 2. Hallazgos diagnósticos del grupo experimental}

\begin{tabular}{lc}
\hline Patologías & Total \\
\hline Sulcus vocalis & 7 \\
Disfonía funcional & 6 \\
Lesión cordal combinada & 10 \\
Masa fibrosa subepitelial & 6 \\
Nódulos vocales & 14 \\
\hline Parálisis cordal & 3 \\
\hline Quiste cuerda vocal & 5
\end{tabular}

El análisis de confiabilidad test-retest para la versión chilena del pVHI total y las diferentes subescalas se muestra en la Tabla 5. El puntaje de correlación de Pearson fue casi perfecto para cada grupo en el pVHI total y en cada una de sus tres subescalas.

\section{Discusión}

La voz es un valioso medio de comunicación y expresión, y en los niños constituye un recurso fundamental para su desarrollo emocional y psicosocial. En la actualidad, existe un

Tabla 3. Puntuación total y de las subescalas física, emocional y funcional del pVHI en la versión chilena

\begin{tabular}{lccc}
\hline & Grupo experimental & Grupo control & p-valor \\
Total pVHI & $35,14 \pm 15,8$ & $5,43 \pm 4,9$ & $0,00 *$ \\
Subescala física & $19,45 \pm 6,5$ & $2,43 \pm 3,1$ & $0,00 *$ \\
Subescala emocional & $7,67 \pm 5,9$ & $1,86 \pm 2,3$ & $0,00^{*}$ \\
Subescala funcional & $8,07 \pm 6,4$ & $1,04 \pm 1,3$ & $0,00 *$ \\
\hline pVHI: Pediatric Voice Handicap Index. ${ }^{*} p<0,05$. & &
\end{tabular}

\begin{tabular}{lcc}
$\begin{array}{l}\text { Tabla 4. Coeficiente alpha de Cronbach: valor general y } \\
\text { de las tres subescalas }\end{array}$ \\
\hline pVHI & Ítems & Alpha de Cronbach \\
\hline Total & 23 & 0,931 \\
\hline Funcional & 7 & 0,912 \\
\hline Físico & 9 & 0,925 \\
\hline Emocional & 7 & 0,943
\end{tabular}

\begin{tabular}{lc}
$\begin{array}{l}\text { Tabla 5. Análisis fiabilidad test-retest del pVHI } \\
\text { total y de las subescalas de la versión chilena }\end{array}$ \\
\hline pVHI & Correlación de Pearson \\
\hline Total & 0,95 \\
Funcional & 0,91 \\
Físico & 0,94 \\
Emocional & 0,93 \\
\hline pVHI: Pediatric voice handicap index.
\end{tabular}


interés creciente en el estudio de los trastornos de la voz y su impacto en la calidad de vida de los niños con disfonía.

El pVHI es uno de los instrumentos más utilizados para evaluar el impacto de la disfonía en los niños. Su versión original ha sido traducida y validada a varios idiomas, incluido el español ${ }^{17-22,24}$. Si bien el español es el idioma utilizado en Chile, consideramos necesario realizar una adaptación cultural al español chileno para obtener una versión compatible con las características lingüísticas de nuestro país para lograr un adecuado entendimiento por parte de los padres o cuidadores.

Es importante mencionar que el cuestionario pVHI traducido al español de Chile no pierde validez frente a la versión original y las versiones en idioma español como las de España y Argentina. Nuestro valor de confiabilidad obtenido mediante el coeficiente alfa de Cronbach para el pVHI total fue $\alpha=0,931$. Para las subescalas el valor fue: físico $\alpha=0,925$, funcional $\alpha=0,912$ y emocional $\alpha=0,943$. En cuanto a la versión española, obtuvo los siguientes valores: $\mathrm{pVHI}$ total $\alpha=0,811$, subescala física $\alpha=0,924$, subescala funcional $\alpha=0,612$ y subescala emocional $\alpha=0,428$.
Finalmente, la versión argentina obtuvo: pVHI total $\alpha=0,951$, subescala física $\alpha=0,879$, subescala funcional $\alpha=0,927$ y subescala emocional $\alpha=0,887^{13,17,22}$.

La fiabilidad test-retest se evaluó mediante coeficiente de correlación de Pearson. Los valores obtenidos fueron los siguientes: 0,95 para el pVHI total, 0,94 para la subescala física, 0,91 para la subescala funcional y 0,93 para la subescala emocional. Se informaron hallazgos similares en el estudio de validación del pVHI original, así como en versiones de habla hispana como las de España y Argentina ${ }^{13,17,22}$. La puntuación total del pVHI y la de sus tres subescalas, física, funcional y emocional en el grupo control fueron significativamente inferiores a las observadas en el grupo experimental. En este último, la puntuación media total del pVHI fue de $35,14 \pm 15,8$ y en las subescalas los resultados fueron: físico $19,45 \pm 6,5$; funcional $8,07 \pm 6,4$ y emocional, $7,67 \pm 5,9$. Estos hallazgos están significativamente por encima de la puntuación media del grupo control y son consistentes con los resultados del estudio original de Zur y cols. y con los resultados de las demás validaciones en español (Figura 2) 13,17,22.

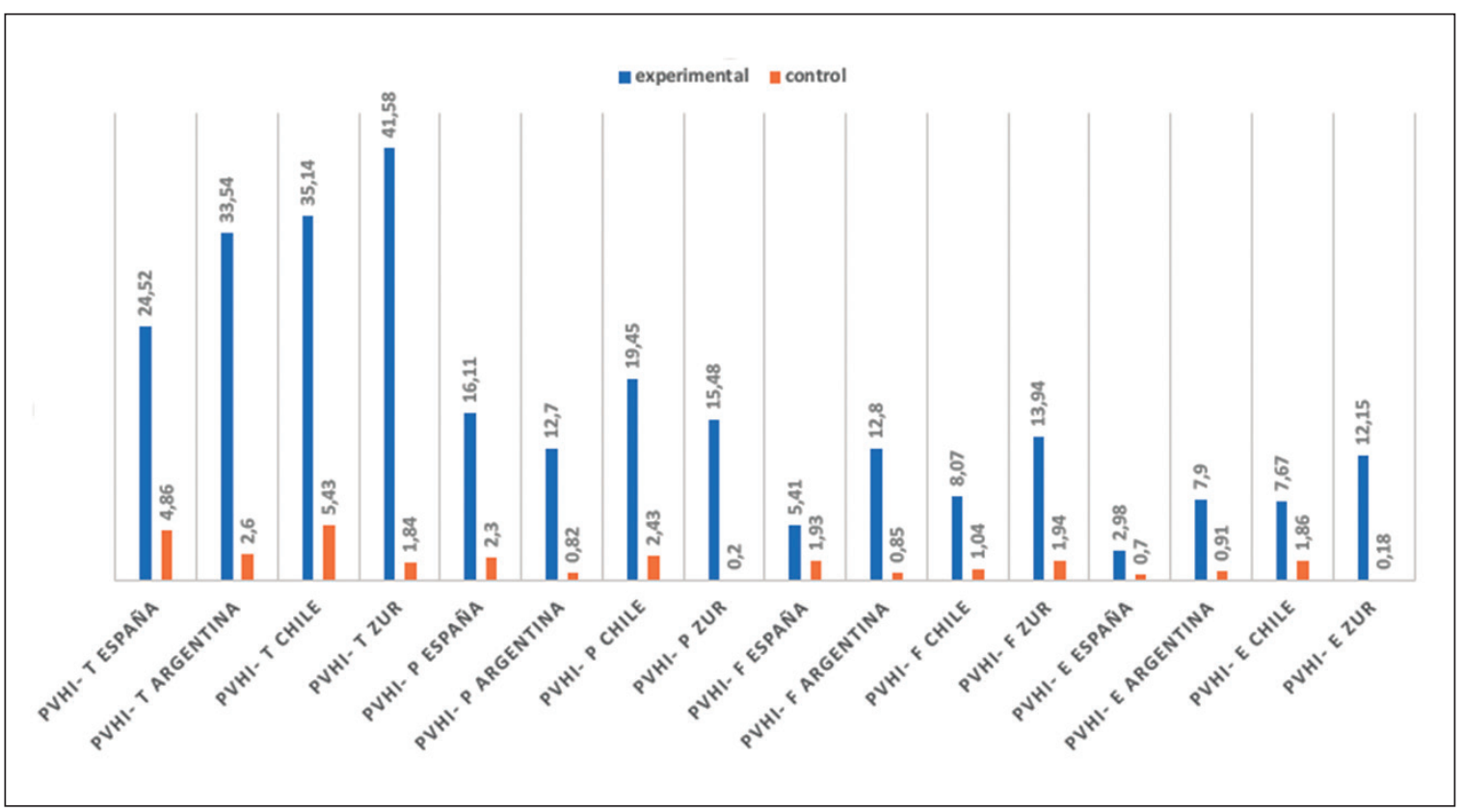

Figura 2. Comparación de los resultados de pVHI entre diversos estudios. 
Nuestras puntuaciones en la subescala física del pVHI fueron más altas que las puntuaciones en las subescalas funcional y emocional de otras investigaciones ${ }^{13,21}$. Si bien los síntomas físicos son los parámetros de percepción más llamativos que los padres asocian directamente con la disfonía, los problemas emocionales y funcionales no se asocian específicamente con un trastorno de la voz. Los niños a menudo no reconocen que su voz está alterada, aunque los padres pueden preocuparse por la ronquera que perciben en ellos. En este contexto, no es infrecuente observar una percepción dispar de la gravedad de la disfonía entre los niños y los padres o cuidadores. El mayor nivel de familiaridad del paciente y asociación con los síntomas físicos de los trastornos de la voz en comparación con los síntomas funcionales y emocionales da como resultado que el dominio físico del pVHI sea el parámetro autopercibido más relevante de los trastornos de la voz ${ }^{24-26}$.

\section{Conclusión}

La versión chilena del cuestionario pVHI presenta un alto grado de validez y confiabilidad. Es una herramienta práctica y sencilla para la apreciación inicial y seguimiento de niños con trastornos de la voz. Por este motivo, recomendamos su uso e implementación como parte del protocolo de evaluación de la voz pediátrica y sus patologías.

\section{Agradecimientos}

A Carola Avendaño San Martín, PhD, Escuela de Pedagogía, Pontificia Universidad Católica de Valparaíso, Chile.

\section{Bibliografía}

1. Vicente J, Hernández-Calvín J, Trinidada A. Enfermedad de las cuerdas vocales: disfonía infantil. An Pediatr Contin. 2008;6:307-312. doi: 10.1016/ S1696-2818(08)74886-8.

2. Coyle S, Weinrich B, Stemple J. Shifts in relative prevalence of laryngeal pathology in a treatment- seeking population. J Voice. 2001;15:424-440. doi: 10.1016/S0892-1997(01)00043-1.

3. Centeno D, Penna M. Caracterización de los pacientes con disfonía evaluados en la Unidad de Voz Pediátrica del Hospital Dr. Luis Calvo Mackenna. Rev Otorrinolaringol Cir Cabeza Cuello. 2019;79:18-24. doi: 10.4067/S0718-48162019000100018.

4. Cavalli L, Cochrane L. Surgical and therapeutic advances in the management of voice problems in children and young people. Curr Opin Otolaryngol Head Neck Surg. 2019;27:178-184. doi: 10.1097/ MOO.0000000000000533.

5. Bhattacharyya N. The prevalence of pediatric voice and swallowing problems in the United States. Laryngoscope. 2015;125:746-750. doi: 10.1002/ lary.24931.

6. Carding P, Roulstone S, Northstone K. The Prevalence of Childhood Dysphonia: A CrossSectional Study. J Voice. 2006;20:623-630. doi: 10.1016/j.jvoice.2005.07.004.

7. Tavares E, Brasolotto A, Santana M, Padovan C, García R. Epidemiological study of dysphonia in 4-12 year-old children. Braz J Otorhinolaryngol. 2011;77:736-746.

8. Mudd P, Noelke C. Vocal fold nodules in children. Curr Opin Otolaryngol Head Neck Surg. 2018;26:426430. doi: 10.1097/MOO.0000000000000496.

9. Dejonckere P, Bradley P, Clemente P, et al. A basic protocol for functional assessment of voice pathology, especially for investigating the efficacy of (phonosurgical) treatments and evaluating new assessment techniques. Eur Arch Oto-RhinoLaryngology. 2001;258:77-82. doi: 10.1007/ s004050000299.

10. Kume M, Ramírez Gorostiza A. Evolución de las técnicas para el estudio de la función vibratoria de las cuerdas vocales. Rev Mex Comun Audiol Otoneurología y Foniatría. 2017;5:7782.

11. Mansour J, Amir O, Sagiv D, Alon E, Wolf M, Primov-Fever A. The Accuracy of Preoperative Rigid Stroboscopy in the Evaluation of Voice Disorders in Children. J Voice. 2017; 32: 516.e1-516.e4. doi: 10.1016/j.jvoice.2016.12.013.

12. Olavarría C, Cortez P. Evaluación y diagnóstico de la disfonía en niños. Neumol Pediatr. 2014;9:75-79.

13. Zur K, Cotton S, Kelchner L, Baker S, Weinrich B, Lee L. Pediatric Voice Handicap Index (pVHI): A new tool for evaluating pediatric dysphonia. Int J Pediatr Otorhinolaryngol. 2007;71:77-82. doi: 10.1016/j. ijporl.2006.09.004.

14. Jacobson B, Johnson A, Grywalski C, Silbergleit 
A, Jacobson G. The Voice Handicap Index (VHI): Development and Validation. Am J Speech-Language Pathol. 1997;6:66-69. doi: 10.1044/1058-0360.0603.66.

15. Schwartz S, Cohen S, Dailey S, et al. Clinical practice guideline: Hoarseness (Dysphonia). Otolaryngol Head Neck Surg. 2009;141:S1-S31. doi: 10.1016/j. otohns.2009.06.744.

16. Betances R, Vallés V. Prevalencia de disfonía infantil en el Colegio de Educación Infantil y Primaria "Ensanche" de Teruel. Rev Otorrinolaringol Cir Cabeza Cuello. 2019;79:159-166. doi: 10.4067/s071848162019000200159 .

17. Sanz L, Bau L, Arribas I, Rivera T. Adaptation and validation of Spanish version of the pediatric Voice Handicap Index (P-VHI). Int J Pediatr Otorhinolaryngol. 2015;79:1439-1443. doi: 10.1016/j. ijporl.2015.06.021.

18. Devadas U, Dhanya M, Gunjawate D. Adaptation and validation of the Malayalam pediatric voice handicap index. Int J Pediatr Otorhinolaryngol. 2015;79:14251428. doi: 10.1016/j.ijporl.2015.06.018.

19. Veder L, Pullens B, Timmerman M, Hoeve H, Joosten K, Hakkesteegt M. Reliability and validity of the Dutch pediatric Voice Handicap Index. Int J Pediatr Otorhinolaryngol. 2017;96:15-20. doi: 10.1016/j. ijporl.2017.02.023.

20. Lu D, Huang M, Li Z, et al. Adaptation and validation of Mandarin Chinese version of the pediatric Voice Handicap Index (pVHI). Int J Pediatr
Otorhinolaryngol. 2018;104:19-24. doi: 10.1016/j. ijporl.2017.10.034.

21. Park S, Kwon T, Choi S, et al. Reliability and validity of the Korean version of Pediatric Voice Handicap Index: In school age children. Int J Pediatr Otorhinolaryngol. 2013;77:107-112. doi: 10.1016/j. ijporl.2012.10.006.

22. Carrera Fernández S, Gabaldón P, Wilder F, Preciado $\mathrm{D}$, Rodríguez $\mathrm{H}$. Index of pediatric voice handicap: Translation, transculturalization and validation to Argentinian Spanish. Int J Pediatr Otorhinolaryngol. 2019:127:109663. doi: 10.1016/j.ijporl.2019.109663.

23. Beaton D, Bombadier C, Guillemin F, Ferraz M. Guidelines for the process of cross-cultural adaptation of self-report measures. Spine. 2000;25(24):3186-91.

24. Schindler A, Tiddia C, Ghidelli C, Nerone V, Albera $\mathrm{R}$, Ottaviani F. Adaptation and validation of the Italian pediatric voice handicap index. Folia Phoniatr Logop. 2011;63:9-14. doi: 10.1159/000319730.

25. Hersan R, Behlau M. Behavioral management of pediatric dysphonia. Otolaryngol Clin North Am. 2000;33:1097-1109. doi: 10.1016/S00306665(05)70268-1.

26. Hsiung M, Pai L, Wang H. Correlation between voice handicap index and voice laboratory measurements in dysphonic patients. Eur Arch OtoRhino-Laryngology. 2002;259:97-99. doi: 10.1007/ s004050100405. 